\title{
Mendelian randomization rules out the causal relationship between serum lipids and cholecystitis
}

\author{
Hongqun Yang ${ }^{1 \dagger}$, Lanlan Chen ${ }^{2 \dagger}$, Kaiyu Liư ${ }^{2}$, Chengnan $\mathrm{Li}^{2}$, Haitao Li ${ }^{2}$, Kezhen Xiong ${ }^{2}$, Zehan Li ${ }^{2}$ Chuang Lu ,
} Wei Chen ${ }^{2}$ and Yahui Liu ${ }^{1 *}$

\begin{abstract}
Background: The relationship between serum lipids and cholecystitis is still under investigation. To examine the causal effect of serum lipids on cholecystitis using the Mendelian randomization method.

Methods: We conducted univariable Mendelian randomization (MR) analyses using summary statistics from two independent genome-wide association studies (GWAS) on serum lipids $(n=132,908)$ and cholecystitis $(n=361,194)$. Mainly, the inverse-variance weighted (IVW) method was utilized to combine each SNP's causal estimation, and the MR-Egger was adopted as a complementary method, together with the weighted median. Cochrane's Q value was employed to appraise heterogeneity. The MR-Egger intercept and MR-PRESSO were used to detect the horizontal pleiotropy.

Results: Our univariable results displayed a minor protective effect of serum low-density lipoprotein (LDL) cholesterol $(\mathrm{OR}[95 \% \mathrm{Cl}]=0.9984483[0.9984499,0.9984468] ; p=0.008)$ on cholecystitis. No significant causal effect of total cholesterol (TC) $(\mathrm{OR}[95 \% \mathrm{Cl}]=0.9994228$ [0.9994222, 0.9994233]; $p=0.296)$, triglycerides $(\mathrm{OR}[95 \% \mathrm{Cl}]=0.9990893$ $[0.9990882,0.9990903] ; p=0.238)$ and high-density lipoprotein $(\mathrm{HDL})$ cholesterol $(\mathrm{OR}[95 \% \mathrm{Cl}]=0.9997020$ $[0.9997017,0.9997023] ; p=0.565$ ) was found on cholecystitis.

Conclusion: These findings suggest that LDL cholesterolhas a slight protective effect on cholecystitis, which can be easily affected by confounding factors. TC, triglycerides and HDL cholesterol don't have causal effect on cholecystitis. The protective effect of serum lipids on cholecystitis, though possible, remain less certain.
\end{abstract}

Keywords: Mendelian randomization, Cholecystitis, Serum lipids, Cholesterol, Triglycerides

\section{Introduction}

Cholecystitis is one of the most common diseases of the gallbladder. According to a comprehensive survey, 262,411 hospitalizations occurred in 2000 for cholecystitis [1]. Fortunately, the mortality of cholecystitis is only at $0.6 \%$ [1]. Cholecystitis could be roughly classified as

\footnotetext{
*Correspondence: yahui_edu@163.com

${ }^{\dagger}$ Hongqun Yang and Lanlan Chen have contributed equally to this work

1 Secondary Department of Hepatobiliary and Pancreatic Surgery, The

First Hospital of Jilin University, Changchun 130021, China

Full list of author information is available at the end of the article
}

acute cholecystitis acalculous cholecystitis and chronic cholecystitis. Cholecystectomy is the gold standard treatment of all kinds of cholecystitis. Although it is not a big surgery, it puts a financial and physical burden on patients. Therefore, the prevention of cholecystitis is of vital importance. Many risk factors were reported by the previous study such as obesity, old age, female gender, long-term total parenteral nutrition, diabetes, etc. [2]. However, whether serum lipids are risk factors for cholecystitis is still under investigation. Furthermore, the current results are controversial. For example, in univariable 
analyses conducted by Mohr et al. [3], they found a negative association between high-density lipoprotein (HDL) cholesterol, low-density lipoprotein (LDL) cholesterol, and cholecystitis while a case-control study conducted by Wang et al. [4] reported that HDL cholesterol was positively associated with cholecystitis. Thus, we hope to find out the causal relationship between blood lipids and cholecystitis. However, it is difficult to conduct a randomized control study (RCT) to investigate the causal relationship. Therefore, we carried out a Mendelian randomization analysis to overcome the limitations of the previous study.

Mendelian randomization (MR) is a method using genetic variants to determine whether there exists a causal relationship between the exposure (usually a risk factor) and the outcome $[5,6]$. The random assortment of genetic variants follows the law of Mendelian. As a result, the allocation of exposure for each individual is achieved randomly, which is the fundament of MR study. Numerous MR studies have been published in recent years where some of them even overturned widely accepted opinions. For example, the MR analysis of Holmes et al. [7] ruled out the causal effect of HDL cholesterol on coronary heart disease. MR analysis is a good way to overcome difficulties of conventional research such as confounders, loss of follow-up, time-consuming. The effect of the instrumental variables (IV) lasts for the whole life even before the birth of the individual. It is exactly suitable for the investigation of the lifetime exposure's effect on the target disease. Compared to conventional observational studies, MR analysis is more convincing and reliable.

Here, we used single nucleotide polymorphisms (SNP) associated with TC, HDL cholesterol, LDL cholesterol, and triglyceride as instrument variables to conduct MR analysis. We extracted SNPs from the genome-wide association studies (GWAS) from the GLGC consortium to investigate the effect of blood lipids on cholecystitis [8]. The levels of TC, HDL cholesterol, LDL cholesterol, and triglycerides were treated as the exposure separately in the univariable MR analysis to investigate its causal effect on cholecystitis. Thus, we hope to disentangle the complex relationship between blood lipids and cholecystitis.

\section{Methods}

Data source

Data involved in this study are publicly available. We extracted the exposure data from the Global Lipids Genetics Consortium (GLGC, http://lipidgenetics.org/). This GWAS study contained summary statistics from 45 studies. Among them, 37 studies consisted of individuals of European ancestry $(n=114,230)$. Another 9 studies consisted primarily of individuals with non-European ancestry: two studies of South Asian descent $(n=1516$, $\mathrm{n}=3385)$; two studies of East Asian descent $(\mathrm{n}=1771$, $\mathrm{n}=7044$ ); five studies of recent African ancestry, from Uganda $(n=1687)$, from the Caribbean $(n=426)$, and the United States $(n=1614, n=397, n=838)$. The exposure data was given in per SD unit. To identify outlier studies, average standard errors for association statistics from each study were plotted against the study sample size. Allele frequencies were scrutinized to guarantee all analyses using the same strand assignment of alleles. Reported statistics and allelic effects were consistent with published findings for known loci. Genomic control values for study-specific analyses were $<1.20$. Variants whose minor alleles were observed $<7$ times were excluded. Phenotype distribution, proportion of phenotype variance explained by instruments were provided in Table 1.

The summary statistics associated with cholecystitis were extracted from the UK biobank (www.nealelab. is/uk-biobank). They filtered from 487,409 individuals down to 337,199 individuals. Individuals included in the GWAS analysis of the UK biobank were restricted to European descent. Individuals who met any of the following criteria were excluded: (1) closely related individuals, (2) individuals with sex chromosome aneuploidies, (3) individuals who had withdrawn consent from the UK biobank study. Over 92 million imputed autosomal SNPs were available for the GWAS analysis. SNPs with minor allele frequency $>0.1 \%$ and Hardy-Weinberg equilibrium

Table 1 The descriptive statistics of instrument variable and phenotype distribution

\begin{tabular}{|c|c|c|c|c|c|c|}
\hline \multicolumn{6}{|c|}{ Descriptive statistics of instrument variable } & \multirow{2}{*}{$\begin{array}{l}\text { Publication } \\
\text { for } \\
\text { instruments }\end{array}$} \\
\hline Phenotype & Mean \pm SD & Units & $\begin{array}{l}\text { Variance } \\
\text { explained }\end{array}$ & Sample size & Consortium & \\
\hline Cholesterol & $213.28 \pm 42.6$ & $\mathrm{SD}(\mathrm{mg} / \mathrm{dL})$ & 0.16 & 187,365 & GLGC & Willer et al. [8] \\
\hline HDL-cholesterol & $53.3 \pm 15.5$ & $\mathrm{SD}(\mathrm{mg} / \mathrm{dL})$ & 0.17 & 187,167 & GLGC & Willer et al. [8] \\
\hline LDL-cholesterol & $133.6 \pm 38.0$ & $\mathrm{SD}(\mathrm{mg} / \mathrm{dL})$ & 0.15 & 173,082 & GLGC & Willer et al. [8] \\
\hline Triglycerides & $140.85 \pm 87.8$ & $\mathrm{SD}(\mathrm{mg} / \mathrm{dL})$ & 0.13 & 177,861 & GLGC & Willer et al. [8] \\
\hline
\end{tabular}


(HWE) $p$ value $>1 \times 10^{-10}$ were included. Finally, 10.8 million SNPs remained for analysis.

\section{IV selection (F statistics)}

All the SNPs were tested for association with the trait of interest at a genome-wide significant level $\left(p<5 \times 10^{-8}\right)$. SNPs missing in summary statistics of outcome were removed. For the sake of reducing the missing SNPs we selected proxy SNPs (European populations) in high LD $\left(r^{2}>0.8\right)$. We excluded the weak instrument variable. The weak instrument variable was defined as the F statistic less than 10 . The F statistic is usually quoted as a measure of the strength of an instrument variable. SNPs that failed to pass the leave-one-out analysis were eliminated. All the SNPs were scrutinized for linkage disequilibrium (LD) according to the criterion as $\mathrm{r}^{2}<0.01$, kilobase $(\mathrm{kb})>10,000$, clumping threshold $<5 \times 10^{-8}$. Palindromic SNPs were excluded from this research. MRPRESSO global test, outlier test, and distortion test were conducted to identify and remove SNPs with horizontal pleiotropy. Compared to other tests of pleiotropy, all three tests of MR-PRESSO have higher power to detect horizonal pleiotropy except for the perfectly overlapping samples [9]. After series of strict filtration the rest of SNPs were regarded as qualified IVs.

\section{Main design}

Mendelian randomization relies on three assumptions (Fig. 1): (1) the instrument variable (genetic variant) is associated with the risk factor; (2) the instrument variable is not associated with confounders; (3) the instrument variable influences the outcome only by the risk factor. A genetic variant satisfying these assumptions is known as an instrumental variable. SNPs involved in this study could easily fit in assumption 1 . We extracted SNPs related to TC, HDL cholesterol, LDL cholesterol and triglycerides as instrumental variables while the SNPs related to cholecystitis were treated as the outcome. With a SNP used as an IV and a biniary outcome, assuming all associations are log-linear, the causal effect of the exposure on the outcome can be estimated as the ratio of the change in the outcome per additional variant allele divided by the change in the risk factor per additional variant allele [10]. Analyses were performed using $\mathrm{R}$, version 4.0.3 (http: //www.r-project.org). $\mathrm{R}$ package 'TwoSampleMR' and 'MRPRESSO' were employed in this study. The $\mathrm{R}$ code is provided in this Github repository (https://github.com/YangHQ638/MR-research).

\section{Statistical analysis}

Many specific methods of MR analysis are available. We adopted the following three methods: inverse-variance weighted (IVW), MR-Egger, and weighted median. IVW is the main method adopted in both MR analysis and multivariable MR analysis. IVW estimation is the inverse variance weighted combination of ratio estimates [11] where the random effect model was adopted if there was heterogeneity. Wald ratio is used to obtain a ratio from each SNP. MR-Egger can test and estimate the causal effect. It can also test the directional pleiotropy, making

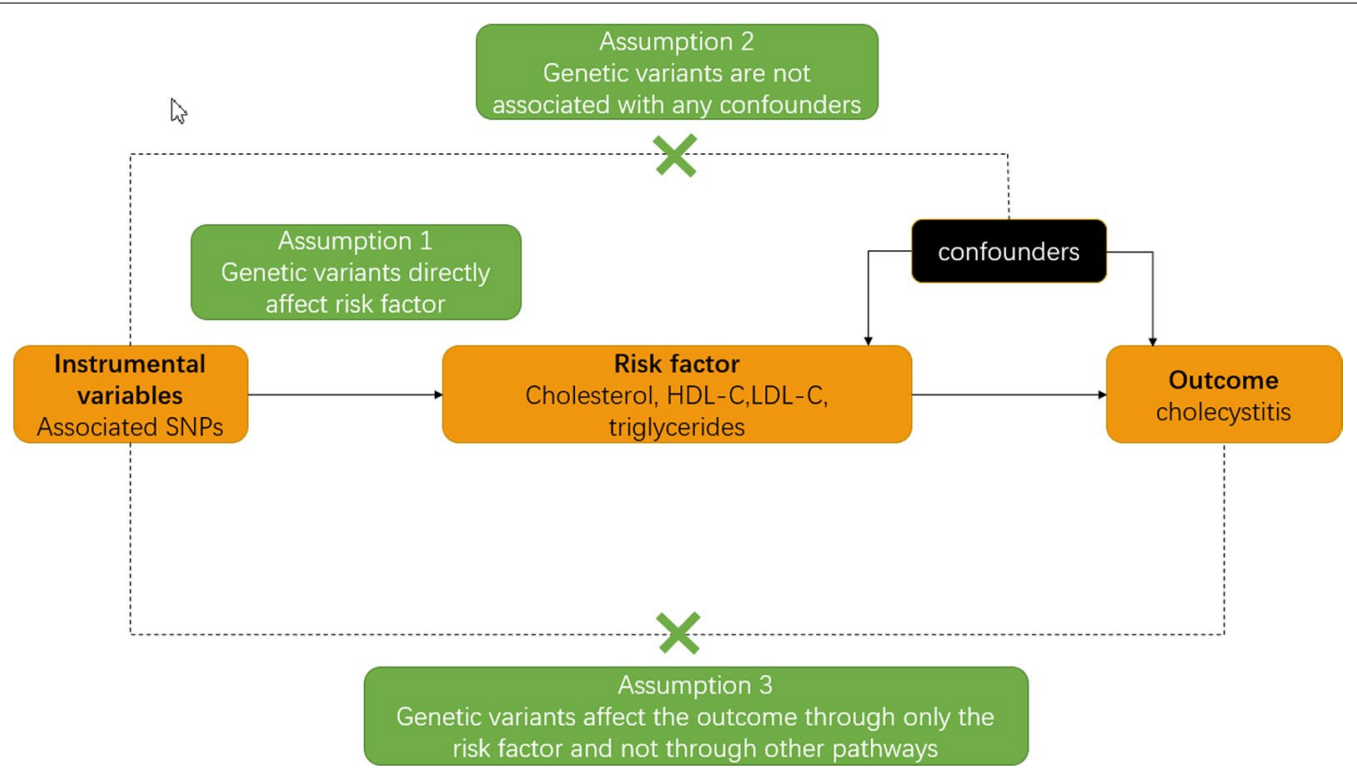

Fig. 1 Three principal assumption of MR analysis. Note: The dashed lines represent the potential causal relationship between variables that would represent violations of the MR assumptions 
it preferred in the case of pleiotropy. The estimation of MR-Egger is close to that of IVW when the intercept term of MR Egger regression is close to 0 [12]. Compared to IVW (fixed-effect model), the weighted median method could correctly estimate the causality when some of the instrumental variables are invalid [12]. Thus, it is complementary to MR Egger. We have adopted all methods for all exposures. The statistical power of this study was tested by an online tool (https://cnsgenomics.shiny apps.io/mRnd/). To address the issue of multiple testing, results were considered statistically significant at the 0.05 level after a false discovery rate approach of the significance level. We conducted a Cochran Q test to examine the heterogeneity of these analyses. We also tested the pleiotropy by collecting the intercept values from MR Egger regression. The leave-one-out sensitivity analysis was performed to test the robustness of this study.

\section{Data visualization}

We made a scatter plot for the result of each exposure. Different lines of scatter plots represent the causal estimation of different methods. Results of sensitivity analysis were visualized by a leave-one-out plot. The pooled result of all SNPs was shown in the forest plot. Biased was tested by funnel plot [13].

\section{Result \\ IV Validation}

The study identified 111 SNPs associated with serum TC level, 123 SNPs associated with HDL cholesterol level, 78 SNPs associated with LDL cholesterol level, and 71 SNPs associated with triglycerides level. All the SNPs were associated with the trait of interest at a genome-wide significant level $\left(p<5^{*} 10^{-8}\right)$. Since the summary statistics were missing in the outcome, 2 SNPs related to TC and 2 SNPs related to LDL cholesterol were removed. None of the SNPs was weak instrumental variable (F statistics $<10) .16$ SNPs associated with LDL cholesterol were omitted by leave-one-out analysis. A total of 5 palindromic SNPs were excluded. Due to pleiotropy, we removed 3 SNPs related to TC and 2 SNPs related to LDL in the MR-PRESSO test. Excluded SNPs, numbers of identified SNPs, were showed in Table 2. Specific identified SNPs of each phenotype were provided in the Additional materials [see Additional file 1].

\section{Main results}

The causal estimations of MR analysis were provided in Table 3. The minor causal effect of LDL cholesterol on cholecystitis was demonstrated by IVW (multiplicative random effect model) (OR $[95 \% \mathrm{CI}]=0.9984483$ $[0.9984499,0.9984468] ; p=0.008)$. There was an evidence of heterogeneity $(\mathrm{Q}=116.489, p$ value $=0.004)$.
Table 2 Excluded SNPs, numbers of identified instrumental SNPS

\begin{tabular}{|c|c|c|c|c|c|}
\hline \multirow[t]{2}{*}{ Phenotype } & \multicolumn{4}{|c|}{ Various types of SNPs excluded } & \multirow[t]{2}{*}{ N SNP } \\
\hline & $\begin{array}{l}\text { Miss in } \\
\text { outcome }\end{array}$ & Pleiotropy & $\begin{array}{l}\text { Leave- } \\
\text { one- } \\
\text { out }\end{array}$ & $\begin{array}{l}\text { Palindromic } \\
\text { structure }\end{array}$ & \\
\hline Cholesterol & 2 & 3 & 2 & 2 & 111 \\
\hline $\begin{array}{l}\text { HDL-choles- } \\
\text { terol }\end{array}$ & 0 & 0 & 0 & 2 & 123 \\
\hline $\begin{array}{l}\text { LDL-choles- } \\
\text { terol }\end{array}$ & 1 & 0 & 16 & 3 & 81 \\
\hline Triglycerides & 0 & 0 & 0 & 1 & 71 \\
\hline
\end{tabular}

NSNP number of identified SNPs

No evidence was found about pleiotropy (egger intercept $=-2.1 \times 10^{-5}, p$ value $=0.659$ ). Although it was not significant at the level of $p=0.05$, a minor protective effect was observed in TC (OR [95\% CI] $=0.9994228$ $[0.9994222,0.9994233] ; p=0.296)$, triglycerides (OR [95\% $\mathrm{CI}]=0.9990893[0.9990882,0.9990903] ; p=0.238)$ and HDL cholesterol (OR $[95 \% \mathrm{CI}]=0.9997020[0.9997017$, 0.9997023 ]; $p=0.565)$. Heterogeneity was detected in HDL cholesterol $(\mathrm{Q}=188.737, p$ value $=0.000)$ and triglycerides $(\mathrm{Q}=96.010, p$ value $=0.021)$ while it was not detected in $\mathrm{TC}(\mathrm{Q}=135.009, p$ value $=0.053)$. The MR-Egger intercept suggested there was no evidence of pleiotropy in TC (egger intercept $=4.06 \times 10^{-5}$, $p$ value $=0.335), \quad$ HDL cholesterol (egger intercept $=4.46 \times 10^{-5}, p$ value $\left.=0.319\right)$, and triglycerides (egger intercept $=6.55 \times 10^{-5}, p$ value $\left.=0.168\right)$. The statistical power of these four exposures was relatively low (TC: 0.08, HDL cholesterol: 0.10, LDL cholesterol: 0.08, triglycerides: 0.08). A Scatter plot of MR results was shown in Fig. 2. Forest plots of each SNP were provided in the Additional materials [see Additional file 2].

\section{Sensitivity analysis}

The funnel plots showed the extent of heterogeneity among the individual Wald ratio estimates. The forest plots displayed the effect estimates of each SNP. The leave-one-out plots showed each SNP was robust in MR analysis. The funnel plots, forest plots and leave-oneout plots were provided in the Additional materials [see Additional file 2].

\section{Discussion}

We conducted univariable MR analyses to investigate the causal effect of serum lipids on cholecystitis. The current study found that LDL cholesterol was protective factors against cholecystitis but such protective effect may be subtle because odds ratios were close to 1 . The results of HDL cholesterolTC and triglycerides were not 
Table 3 The effect estimates, test of heterogeneity and test of pleiotropy of exposure on cholecystitis

\begin{tabular}{|c|c|c|c|c|c|c|c|c|c|}
\hline & \multirow{2}{*}{$\begin{array}{l}\text { MR } \\
\text { methodology }\end{array}$} & \multicolumn{4}{|c|}{ Effect estimates on cholecystitis } & \multicolumn{2}{|c|}{ Test of heterogeneity } & \multirow{2}{*}{$\begin{array}{l}\text { Test of } \\
\text { pleiotropy } \\
\text { MR-Egger } \\
\text { intercept }\end{array}$} & \multirow[t]{2}{*}{$p$ value } \\
\hline & & OR & Lower & Upper & $p$ value & Cochrane $\mathrm{Q}$ test & $p$ value & & \\
\hline \multirow[t]{4}{*}{ Total cholesterol } & $\begin{array}{l}\text { Inverse variance } \\
\text { weighted (multi- } \\
\text { plicative random } \\
\text { effects) }\end{array}$ & 0.9994228 & 0.9994222 & 0.9994233 & 0.2960 & 135.009 & 0.053 & & \\
\hline & MR Egger & 0.9987183 & 0.9987161 & 0.9987205 & 0.1893 & 133.856 & 0.053 & 0.0000406 & 0.335 \\
\hline & $\begin{array}{l}\text { Inverse variance } \\
\text { weighted (fixed } \\
\text { effects) }\end{array}$ & 0.9994228 & 0.9994223 & 0.9994232 & 0.2347 & 135.009 & 0.053 & & \\
\hline & $\begin{array}{l}\text { Weighted } \\
\text { median }\end{array}$ & 0.9994726 & 0.9994719 & 0.9994733 & 0.5640 & & & & \\
\hline \multirow[t]{4}{*}{$\mathrm{HDL}-\mathrm{C}$} & $\begin{array}{l}\text { Inverse variance } \\
\text { weighted (multi- } \\
\text { plicative random } \\
\text { effects) }\end{array}$ & 0.9997020 & 0.9997017 & 0.9997023 & 0.5650 & 188.737 & 0.000 & & \\
\hline & MR Egger & 0.9988892 & 0.9988871 & 0.9988913 & 0.2510 & 187.188 & 0.000 & 0.0000446 & 0.319 \\
\hline & $\begin{array}{l}\text { Inverse variance } \\
\text { weighted (fixed } \\
\text { effects) }\end{array}$ & 0.9997020 & 0.9997018 & 0.9997023 & 0.4740 & 188.737 & 0.000 & & \\
\hline & $\begin{array}{l}\text { Weighted } \\
\text { median }\end{array}$ & 0.9996111 & 0.9996106 & 0.9996117 & 0.5870 & & & & \\
\hline \multirow[t]{4}{*}{ LDL-C } & $\begin{array}{l}\text { Inverse variance } \\
\text { weighted (multi- } \\
\text { plicative random } \\
\text { effects) }\end{array}$ & 0.9984483 & 0.9984499 & 0.9984468 & 0.0080 & 116.489188 & 0.004851838 & & \\
\hline & MR Egger & 0.9987160 & 0.9987180 & 0.9987140 & 0.1893 & 116.200524 & 0.004109598 & -0.000021 & 0.658974602 \\
\hline & $\begin{array}{l}\text { Inverse variance } \\
\text { weighted (fixed } \\
\text { effects) }\end{array}$ & 0.9984483 & 0.9984496 & 0.9984471 & 0.0008 & 116.489188 & 0.004851838 & & \\
\hline & $\begin{array}{l}\text { Weighted } \\
\text { median }\end{array}$ & 0.9991214 & 0.9991225 & 0.9991203 & 0.3380 & & & & \\
\hline \multirow[t]{4}{*}{ Triglycerides } & $\begin{array}{l}\text { Inverse variance } \\
\text { weighted (multi- } \\
\text { plicative random } \\
\text { effects) }\end{array}$ & 0.9990893 & 0.9990882 & 0.9990903 & 0.2380 & 96.010 & 0.021 & & \\
\hline & MR Egger & 0.9980924 & 0.9980889 & 0.9980958 & 0.1680 & 93.378 & 0.027 & 0.0000655 & 0.168 \\
\hline & $\begin{array}{l}\text { Inverse variance } \\
\text { weighted (fixed } \\
\text { effects) }\end{array}$ & 0.9990893 & 0.9990884 & 0.9990902 & 0.1360 & 96.010 & 0.021 & & \\
\hline & $\begin{array}{l}\text { Weighted } \\
\text { median }\end{array}$ & 0.9983449 & 0.9983424 & 0.9983474 & 0.1360 & & & & \\
\hline
\end{tabular}

TC total cholesterol, HDL-C HDL cholesterol, LDL-C LDL cholesterol, TG triglycerides, OR odds ratio, upper upper bounds of $95 \%$ confidence interval, lower lower bounds of $95 \%$ confidence interval. $p$ value was adjusted by false discovery rate approach

statistically significant but all of them showed a protective tendency. The causal association of serum lipids with cholecystitis remains possible, but less certain. Statistical power was relatively low, but nevertheless, the protective effect of LDL cholesterol could still be demonstrated in current study. LDL cholesterol and triglycerides showed a similar trend as a protective factor of cholecystitis, which was partially consistent with some of the previous findings. In univariate analyses conducted by Mohr et al. [3], they found an inverse relationship between HDL cholesterol, LDL cholesterol, and cholecystitis but they also found LDL cholesterol and triglycerides were positively related while HDL cholesterol was not related to cholecystitis in multiple regression. A case-control study conducted by Wang et al. [4] reported that LDL cholesterol was reversely related while HDL cholesterol was positively related. Wang et al. also found a low level of TC increased the risk of gallstone disease [4]. Gallstones can directly induce cholecystitis. Several reports have shown that cholesterol played an important role in the process 

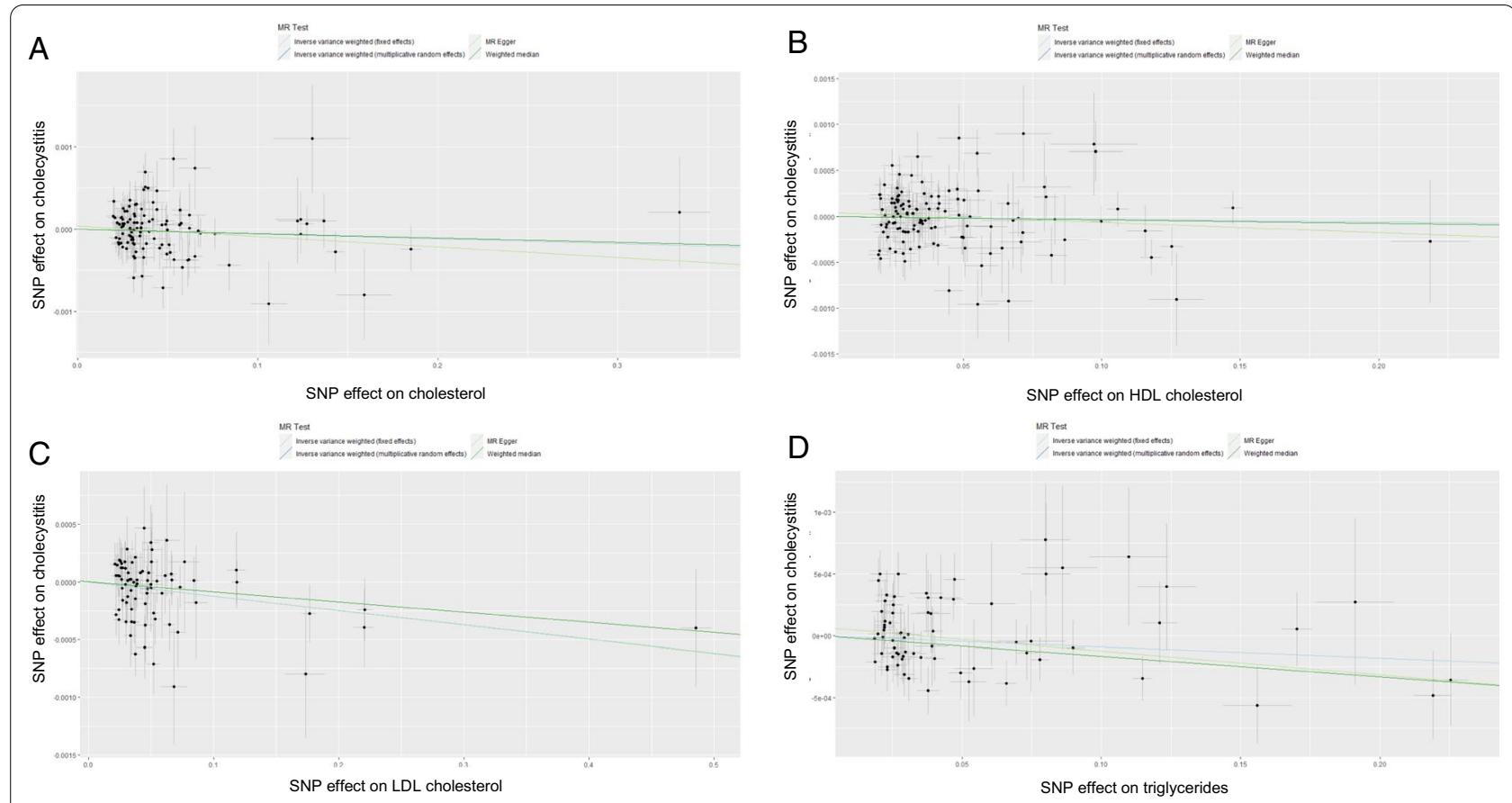

Fig. 2 Scatter plots of different lipids. Note: Scatter plots showed the causal effect of exposure on cholecystitis. A Effect of cholesterol, B effect of $\mathrm{HDL}$ cholesterol, $\mathbf{C}$ effect of $\mathrm{LDL}$ cholesterol, $\mathbf{D}$ effect of triglycerides

of gallstone formation $[3,14,15]$. Jiang et al. also reported that patients with gallstone disease had lower serum TC [16]. However, Another case-control study failed to find an association between HDL cholesterol and gallbladder disease [17]. The reason why there are different opinions is that the protective effect of specific serum lipid is really small (but they do exist which is demonstrated in this research). Even some confounding factors are enough to affect the result.

A possible explanation for the protective effect might be the differences in cholesterol transport and secretion. Clinical studies have demonstrated an inverse relationship between serum HDL cholesterol level and bile cholesterol saturation [18]. Lithogenic bile with supersaturated cholesterol is the initial event of the pathogenesis of gallstones and chronic cholecystitis [19, 20]. Supersaturated concentrations of biliary cholesterol cause abnormalities in the mucosa and muscle layers of the gallbladder [19]. They are the results of increase transport of cholesterol across the gallbladder mucosa due to the high concentration in gallbladder [20], which is the result of increasing cholesterol transport from serum to gallbladder. This phenomenon may cause the decrease of serum cholesterol and increase of supersaturated cholesterol biliary simultaneously. Analysis of inbred mice also showed a similar result [21]. Some biochemical analyses have found many molecules related to the transport of cholesterol. SR-BI (scavenger receptor class B type I) is an important determinant of plasma-to-bile transport of HDL cholesterol. Mice with overexpression of SR-BI enhanced biliary cholesterol levels while a decrease of serum cholesterol level was observed in SR-BI deficiency mice [22-25]. SR-BI might upregulate in cholecystitis patients which needs further research to verify. Juvonen et al. reported TaqBI CEPT gene polymorphism, which is correlated with lower serum TC, was associated with cholesterol gallstone disease [14]. It has been demonstrated that gallstone patients secret more cholesterol than normal people $[19,26]$. Gallstones could directly lead to cholecystitis. The absolute decrease in serum TC might be a crucial factor in determining cholesterol saturation in bile [27]. However, a long-term high cholesterol diet might cause an increase in serum TC and indirectly increased the secretion of cholesterol [28]. Nevertheless, the way of instrumental variable affect exposure was not the same as the way of diet. So, if we could exclude people with high cholesterol diet, then the result would be clearer. This might be the reason that odds ratio of serum lipids was near to 1 . Along with instrumental variables, confounders and environmental factors may co-influence the final result. It is important to conduct a stratified analysis for future research.

Another explanation for our result might be that some of these lipids accelerate gallbladder emptying. Hopman 
et al. [29] have demonstrated that long-chain triglycerides increased the concentration of plasma cholecystokinin (CCK), which could enhance gallbladder motility while medium-chain triglycerides were not. This might explain that the protective effect of triglycerides was very small. Our results have demonstrated that triglyceride was a minor protective factor. There might be some unknown interactions between different types of cholesterol. Further analyses need to find out the effect of different types of cholesterol and the effect of triglycerides on the gallbladder.

There were still a few drawbacks to our study. Firstly, canalization might have an impact on our result. Due to the whole life-span exposure to low levels of plasma lipids, the body might emerge a negative feedback mechanism to compensate. Secondly, because the instrument variable only could slightly affect the exposure, our result was based on extrapolation. Thirdly, our study was based on a mixed population. Because of different races, cultures, diets, and climate, a study based on a specific population might more persuasive. Moreover, recent epidemiological studies reported women are at a higher risk than men [30], while others believed not [4]. Due to limitations of the Mendelian randomization study, we didn't investigate the differences between genders. Further studies should be based on a stratified population to overcome this difficulty. Finally, limited by the data source, the statistical power of this research was quite low, hence typeIIerror might occurs in this research. However, it didn't affect the conclusion that LDL cholesterol was a protective factor against cholecystitis.

Furthermore, previous studies have investigated or stated hypotheses a few underlying mechanisms of lipids affecting cholecystitis. Measuring serum lipids is much easier than measuring cholesterol in the gallbladder in clinical practice. Future studies should not only focus on the relationship between cholesterol saturation and cholecystitis but also investigate the relationship between serum lipids and cholecystitis.

In conclusion, the minor protective effect of LDL cholesterol has been demonstrated in this MR analysis. The protective effect of HDL cholesterol, triglycerides and TC on cholecystitis were also found but not statistically significant in this study. However, the protective effect demonstrated in this study is negligible. The protective effect of serum lipids on cholecystitis, though possible, remain less certain.

\section{Abbreviations}

LDL: Low-density lipoprotein; HDL: High-density lipoprotein; TC: Total cholesterol; MR: Mendelian randomization; SNP: Single nucleotide polymorphisms; GWAS: Genome-wide association studies; HWE: Hardy-Weinberg equilibrium.

\section{Supplementary Information}

The online version contains supplementary material available at https://doi. org/10.1186/s12920-021-01082-y.

Additional file 1. Identified SNPS. Identified SNPS for each exposure (TC, HDL cholesterol, LDL cholesterol, triglycerides).

Additional file 2. None. Funnel plots, forest plots and leave-one-out plots for each exposure (TC, HDL cholesterol, LDL cholesterol, triglycerides).

\section{Acknowledgements}

Support in writing manuscript and data analysis was provided by Bai Ji and Qiong Yu. We thank C. J. Willer et al. who kindly provided the GWAS data for our work.

\section{Authors' contributions}

$\mathrm{HY}$ and $\mathrm{YL}$ designed this research; $\mathrm{CL}, \mathrm{HL}$ and $\mathrm{KL}$ did the data acquisition; $\mathrm{HY}$ and $L C$ conducted the statistical analyses; HY, LC, ZL and KX wrote the first draft of the manuscript; $L C, H Y, C L$ and WC revised the manuscript. All authors agreed with the manuscript submission. YL gave the final approval for the manuscript submission. All authors read and approved the final manuscript.

\section{Funding}

This research did not receive any specific grant from funding agencies in the public, commercial, or not-for-profit sectors.

\section{Availability of data and materials}

The data used to support the findings of this study are included within the article.

\section{Declarations}

Ethics approval and consent to participate Not applicable.

\section{Consent for publication}

All the authors have consented for publication.

\section{Competing interests}

The authors declare that they have no competing interest.

\section{Author details}

${ }^{1}$ Secondary Department of Hepatobiliary and Pancreatic Surgery, The First Hospital of Jilin University, Changchun 130021, China. ${ }^{2}$ Clinical Medical School, Jilin University, Changchun 130021, Jilin, China.

Received: 8 July 2021 Accepted: 7 September 2021

Published online: 17 September 2021

\section{References}

1. Shaffer EA. Gallstone disease: epidemiology of gallbladder stone disease. Best Pract Res Clin Gastroenterol. 2006;20(6):981-96.

2. Elwood DR. Cholecystitis. Surg Clin North Am. 2008;88(6):1241-52, viii.

3. Mohr GC, Kritz-Silverstein D, Barrett-Connor E. Plasma lipids and gallbladder disease. Am J Epidemiol. 1991;134(1):78-85.

4. Wang J, Shen S, Wang B, Ni X, Liu H, Ni X, Yu R, Suo T, Liu H. Serum lipid levels are the risk factors of gallbladder stones: a population-based study in China. Lipids Health Dis. 2020;19(1):50.

5. Emdin CA, Khera AV, Kathiresan S. Mendelian randomization. JAMA. 2017;318(19):1925-6.

6. Smith GD, Ebrahim S. "Mendelian randomization": can genetic epidemiology contribute to understanding environmental determinants of disease? Int J Epidemiol. 2003;32(1):1-22.

7. Holmes MV, Asselbergs FW, Palmer TM, Drenos F, Lanktree MB, Nelson CP, Dale CE, Padmanabhan S, Finan C, Swerdlow DI, et al. Mendelian 
randomization of blood lipids for coronary heart disease. Eur Heart J. 2015;36(9):539-50.

8. Willer CJ, Schmidt EM, Sengupta S, Peloso GM, Gustafsson S, Kanoni S, Ganna A, Chen J, Buchkovich ML, Mora S, et al. Discovery and refinement of loci associated with lipid levels. Nat Genet. 2013:45(11):1274-83.

9. Verbanck M, Chen CY, Neale B, Do R. Detection of widespread horizontal pleiotropy in causal relationships inferred from Mendelian randomization between complex traits and diseases. Nat Genet. 2018;50(5):693-8.

10. Thomas DC, Conti DV. Commentary: the concept of "Mendelian randomization." Int J Epidemiol. 2004;33(1):21-5.

11. Burgess S, Butterworth A, Thompson SG. Mendelian randomization analysis with multiple genetic variants using summarized data. Genet Epidemiol. 2013;37(7):658-65.

12. Burgess S, Thompson SG. Interpreting findings from Mendelian randomization using the MR-Egger method. Eur J Epidemiol. 2017;32(5):377-89.

13. Egger M, Davey Smith G, Schneider M, Minder C. Bias in meta-analysis detected by a simple, graphical test. BMJ. 1997;315(7109):629-34.

14. Juvonen T, Savolainen MJ, Kairaluoma MI, Lajunen LH, Humphries SE, Kesäniemi YA. Polymorphisms at the apoB, apoA-I, and cholesteryl ester transfer protein gene loci in patients with gallbladder disease. J Lipid Res. 1995;36(4):804-12.

15. Fuchs M, Lammert F, Wang DQ, Paigen B, Carey MC, Cohen DE. Sterol carrier protein 2 participates in hypersecretion of biliary cholesterol during gallstone formation in genetically gallstone-susceptible mice. Biochem $\mathrm{J}$. 1998;336(Pt 1):33-7.

16. Jiang ZY, Han TQ, Suo GJ, Feng DX, Chen S, Cai XX, Jiang ZH, Shang J, Zhang Y, Jiang Y, et al. Polymorphisms at cholesterol 7alpha-hydroxylase, apolipoproteins $B$ and $E$ and low density lipoprotein receptor genes in patients with gallbladder stone disease. World J Gastroenterol. 2004;10(10):1508-12.

17. Scragg RK, Calvert GD, Oliver JR. Plasma lipids and insulin in gall stone disease: a case-control study. Br Med J (Clin Res Ed). 1984;289(6444):521-5.

18. Thornton JR, Heaton KW, Macfarlane DG. A relation between high-density-lipoprotein cholesterol and bile cholesterol saturation. Br Med J (Clin Res Ed). 1981;283(6303):1352-4.

19. van Erpecum KJ, Wang DQ, Moschetta A, Ferri D, Svelto M, Portincasa P, Hendrickx JJ, Schipper M, Calamita G. Gallbladder histopathology during murine gallstone formation: relation to motility and concentrating function. J Lipid Res. 2006;47(1):32-41.

20. Jacyna MR, Ross PE, Bakar MA, Hopwood D, Bouchier IA. Characteristics of cholesterol absorption by human gall bladder: relevance to cholesterolosis. J Clin Pathol. 1987;40(5):524-9.
21. Khanuja B, Cheah YC, Hunt M, Nishina PM, Wang DQ, Chen HW, Billheimer JT, Carey MC, Paigen B. Lith1, a major gene affecting cholesterol gallstone formation among inbred strains of mice. Proc Natl Acad Sci U S A. 1995;92(17):7729-33.

22. Mardones P, Quiñones V, Amigo L, Moreno M, Miquel JF, Schwarz M, Miettinen HE, Trigatti B, Krieger M, VanPatten S, et al. Hepatic cholesterol and bile acid metabolism and intestinal cholesterol absorption in scavenger receptor class B type I-deficient mice. J Lipid Res. 2001;42(2):170-80.

23. Trigatti B, Rayburn H, Viñals M, Braun A, Miettinen H, Penman M, Hertz M, Schrenzel M, Amigo L, Rigotti A, et al. Influence of the high density lipoprotein receptor SR-BI on reproductive and cardiovascular pathophysiology. Proc Natl Acad Sci U S A. 1999;96(16):9322-7.

24. Van Eck M, Twisk J, Hoekstra M, Van Rij BT, Van der Lans CA, Bos IS, Kruijt JK, Kuipers F, Van Berkel TJ. Differential effects of scavenger receptor B deficiency on lipid metabolism in cells of the arterial wall and in the liver. J Biol Chem. 2003;278(26):23699-705.

25. Wang DQ, Carey MC. Susceptibility to murine cholesterol gallstone formation is not affected by partial disruption of the HDL receptor SR-BI. Biochim Biophys Acta. 2002;1583(2):141-50.

26. Salen G, Nicolau G, Shefer S, Mosbach EH. Hepatic cholesterol metabolism in patients with gallstones. Gastroenterology. 1975;69(3):676-84.

27. von Bergmann $\mathrm{K}$, Leiss $\mathrm{O}$. Effect of short-term treatment with bezafibrate and fenofibrate on biliary lipid metabolism in patients with hyperlipoproteinaemia. Eur J Clin Investig. 1984;14(2):150-4.

28. Kakimoto T, Kanemoto H, Fukushima K, Ohno K, Tsujimoto H. Effect of a high-fat-high-cholesterol diet on gallbladder bile acid composition and gallbladder motility in dogs. Am J Vet Res. 2017;78(12):1406-13.

29. Hopman WP, Jansen JB, Rosenbusch G, Lamers CB. Effect of equimolar amounts of long-chain triglycerides and medium-chain triglycerides on plasma cholecystokinin and gallbladder contraction. Am J Clin Nutr. 1984;39(3):356-9.

30. Behar J, Mawe GM, Carey MC. Roles of cholesterol and bile salts in the pathogenesis of gallbladder hypomotility and inflammation: cholecystitis is not caused by cystic duct obstruction. Neurogastroenterol Motil. 2013;25(4):283-90.

\section{Publisher's Note}

Springer Nature remains neutral with regard to jurisdictional claims in published maps and institutional affiliations.
Ready to submit your research? Choose BMC and benefit from:

- fast, convenient online submission

- thorough peer review by experienced researchers in your field

- rapid publication on acceptance

- support for research data, including large and complex data types

- gold Open Access which fosters wider collaboration and increased citations

- maximum visibility for your research: over $100 \mathrm{M}$ website views per year

At BMC, research is always in progress.

Learn more biomedcentral.com/submissions 